\title{
Cross-Lingual Pre-Training Based Transfer for Zero-Shot Neural Machine Translation
}

\author{
Baijun Ji, ${ }^{\ddagger}$ Zhirui Zhang, ${ }^{\S}$ Xiangyu Duan, ${ }^{\dagger \dagger *}$ Min Zhang, ${ }^{\dagger \ddagger}$ Boxing Chen,,${ }^{\S}$ Weihua Luo ${ }^{\S}$ \\ ${ }^{\dagger}$ Institute of Artificial Intelligence, Soochow University, Suzhou, China \\ $¥$ School of Computer Science and Technology, Soochow University, Suzhou, China \\ $\S$ Alibaba DAMO Academy, Hangzhou, China \\ †bjji@stu.suda.edu.cn, $¥\{$ xiangyuduan, minzhang\}@suda.edu.cn \\ $\S\{$ zhirui.zzr, boxing.cbx, weihua.luowh $\} @$ alibaba-inc.com
}

\begin{abstract}
Transfer learning between different language pairs has shown its effectiveness for Neural Machine Translation (NMT) in low-resource scenario. However, existing transfer methods involving a common target language are far from success in the extreme scenario of zero-shot translation, due to the language space mismatch problem between transferor (the parent model) and transferee (the child model) on the source side. To address this challenge, we propose an effective transfer learning approach based on cross-lingual pre-training. Our key idea is to make all source languages share the same feature space and thus enable a smooth transition for zero-shot translation. To this end, we introduce one monolingual pretraining method and two bilingual pre-training methods to obtain a universal encoder for different languages. Once the universal encoder is constructed, the parent model built on such encoder is trained with large-scale annotated data and then directly applied in zero-shot translation scenario. Experiments on two public datasets show that our approach significantly outperforms strong pivot-based baseline and various multilingual NMT approaches.
\end{abstract}

\section{Introduction}

Although Neural Machine Translation (NMT) has dominated recent research on translation tasks (Wu et al. 2016; Vaswani et al. 2017; Hassan et al. 2018), NMT heavily relies on large-scale parallel data, resulting in poor performance on low-resource or zero-resource language pairs (Koehn and Knowles 2017). Translation between these low-resource languages (e.g., Arabic $\rightarrow$ Spanish) is usually accomplished with pivoting through a rich-resource language (such as English), i.e., Arabic (source) sentence is translated to English (pivot) first which is later translated to Spanish (target) (Kauers et al. 2002; de Gispert and Mariño 2006). However, the pivot-based method requires doubled decoding time and suffers from the propagation of translation errors.

One common alternative to avoid pivoting in NMT is transfer learning (Zoph et al. 2016; Nguyen and Chiang 2017; Kocmi and Bojar 2018; Kim et al. 2019) which leverages a high-resource pivot $\rightarrow$ target model (parent) to ini-

\footnotetext{
${ }^{*}$ Corresponding Author. Copyright (c) 2020, Association for the Advancement of Artificial Intelligence (www.aaai.org). All rights reserved.
}

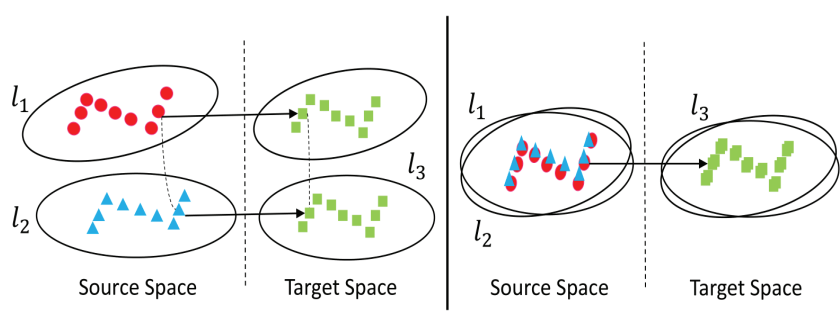

Figure 1: The circle and triangle dots represent source sentences in different language $l_{1}$ and $l_{2}$, and the square dots means target sentences in language $l_{3}$. A sample of translation pairs is connected by the dashed line. We would like to force each of the translation pairs has the same latent representation as the right part of the figure so as to transfer $l_{1} \rightarrow l_{3}$ model directly to $l_{2} \rightarrow l_{3}$ model.

tialize a low-resource source $\rightarrow$ target model (child) that is further optimized with a small amount of available parallel data. Although this approach has achieved success in some low-resource language pairs, it still performs very poorly in extremely low-resource or zero-resource translation scenario. Specifically, Kocmi and Bojar (2018) reports that without any child model training data, the performance of the parent model on the child test set is miserable.

In this work, we argue that the language space mismatch problem, also named domain shift problem (Fu et al. 2015), brings about the zero-shot translation failure in transfer learning. It is because transfer learning has no explicit training process to guarantee that the source and pivot languages share the same feature distributions, causing that the child model inherited from the parent model fails in such a situation. For instance, as illustrated in the left of Figure 1 , the points of the sentence pair with the same semantics are not overlapping in source space, resulting in that the shared decoder will generate different translations denoted by different points in target space. Actually, transfer learning for NMT can be viewed as a multi-domain problem where each source language forms a new domain. Minimizing the discrepancy between the feature distributions of different source languages, i.e., different domains, will ensure the smooth transition between the parent and child models, 
as shown in the right of Figure 1. One way to achieve this goal is the fine-tuning technique, which forces the model to forget the specific knowledge from parent data and learn new features from child data. However, the domain shift problem still exists, and the demand of parallel child data for finetuning heavily hinders transfer learning for NMT towards the zero-resource setting.

In this paper, we explore the transfer learning in a common zero-shot scenario where there are a lot of source $\leftrightarrow$ pivot and pivot $\leftrightarrow$ target parallel data but no source $\leftrightarrow$ target parallel data. In this scenario, we propose a simple but effective transfer approach, the key idea of which is to relieve the burden of the domain shift problem by means of cross-lingual pre-training. To this end, we firstly investigate the performance of two existing cross-lingual pre-training methods proposed by Lample and Conneau (2019) in zero-shot translation scenario. Besides, a novel pre-training method called BRidge Language Modeling (BRLM) is designed to make full use of the source $\leftrightarrow$ pivot bilingual data to obtain a universal encoder for different languages. Once the universal encoder is constructed, we only need to train the pivot $\rightarrow$ target model and then test this model in source $\rightarrow$ target direction directly. The main contributions of this paper are as follows:

- We propose a new transfer learning approach for NMT which uses the cross-lingual language model pre-training to enable a high performance on zero-shot translation.

- We propose a novel pre-training method called BRLM, which can effectively alleviates the distance between different source language spaces.

- Our proposed approach significantly improves zero-shot translation performance, consistently surpassing pivoting and multilingual approaches. Meanwhile, the performance on supervised translation direction remains the same level or even better when using our method.

\section{Related Work}

In recent years, zero-shot translation in NMT has attracted widespread attention in academic research. Existing methods are mainly divided into four categories: pivot-based method, transfer learning, multilingual NMT, and unsupervised NMT.

- Pivot-based Method is a common strategy to obtain a source $\rightarrow$ target model by introducing a pivot language. This approach is further divided into pivoting and pivotsynthetic. While the former firstly translates a source language into the pivot language which is later translated to the target language (Kauers et al. 2002; de Gispert and Mariño 2006; Utiyama and Isahara 2007), the latter trains a source $\rightarrow$ target model with pseudo data generated from source-pivot or pivot-target parallel data (Chen et al. 2017; Zheng, Cheng, and Liu 2017). Although the pivot-based methods can achieve not bad performance, it always falls into a computation-expensive and parametervast dilemma of quadratic growth in the number of source languages, and suffers from the error propagation problem (Zhu et al. 2013).
- Transfer Learning is firstly introduced for NMT by Zoph et al. (2016), which leverages a high-resource parent model to initialize the low-resource child model. On this basis, Nguyen and Chiang (2017) and Kocmi and Bojar (2018) use shared vocabularies for source/target language to improve transfer learning, while Kim, Gao, and Ney (2019) relieve the vocabulary mismatch by mainly using cross-lingual word embedding. Although these methods are successful in the low-resource scene, they have limited effects in zero-shot translation.

- Multilingual NMT (MNMT) enables training a single model that supports translation from multiple source languages into multiple target languages, even those unseen language pairs (Firat, Cho, and Bengio 2016; Firat et al. 2016; Johnson et al. 2016; Al-Shedivat and Parikh 2019; Aharoni, Johnson, and Firat 2019). Aside from simpler deployment, MNMT benefits from transfer learning where low-resource language pairs are trained together with high-resource ones. However, Gu et al. (2019) point out that MNMT for zero-shot translation easily fails, and is sensitive to the hyper-parameter setting. Also, MNMT usually performs worse than the pivot-based method in zero-shot translation setting (Arivazhagan et al. 2018).

- Unsupervised NMT (UNMT) considers a harder setting, in which only large-scale monolingual corpora are available for training. Recently, many methods have been proposed to improve the performance of UNMT, including using denoising auto-encoder, statistic machine translation (SMT) and unsupervised pre-training (Artetxe et al. 2017; Lample et al. 2018; Ren et al. 2019; Lample and Conneau 2019). Since UNMT performs well between similar languages (e.g., English-German translation), its performance between distant languages is still far from expectation.

Our proposed method belongs to the transfer learning, but it is different from traditional transfer methods which train a parent model as starting point. Before training a parent model, our approach fully leverages cross-lingual pretraining methods to make all source languages share the same feature space and thus enables a smooth transition for zero-shot translation.

\section{Approach}

In this section, we will present a cross-lingual pretraining based transfer approach. This method is designed for a common zero-shot scenario where there are a lot of source $\leftrightarrow$ pivot and pivot $\leftrightarrow$ target bilingual data but no source $\leftrightarrow$ target parallel data, and the whole training process can be summarized as follows step by step:

- Pre-train a universal encoder with source/pivot monolingual or source $\leftrightarrow$ pivot bilingual data.

- Train a pivot $\rightarrow$ target parent model built on the pre-trained universal encoder with the available parallel data. During the training process, we freeze several layers of the pre-trained universal encoder to avoid the degeneracy issue (Howard and Ruder 2018). 


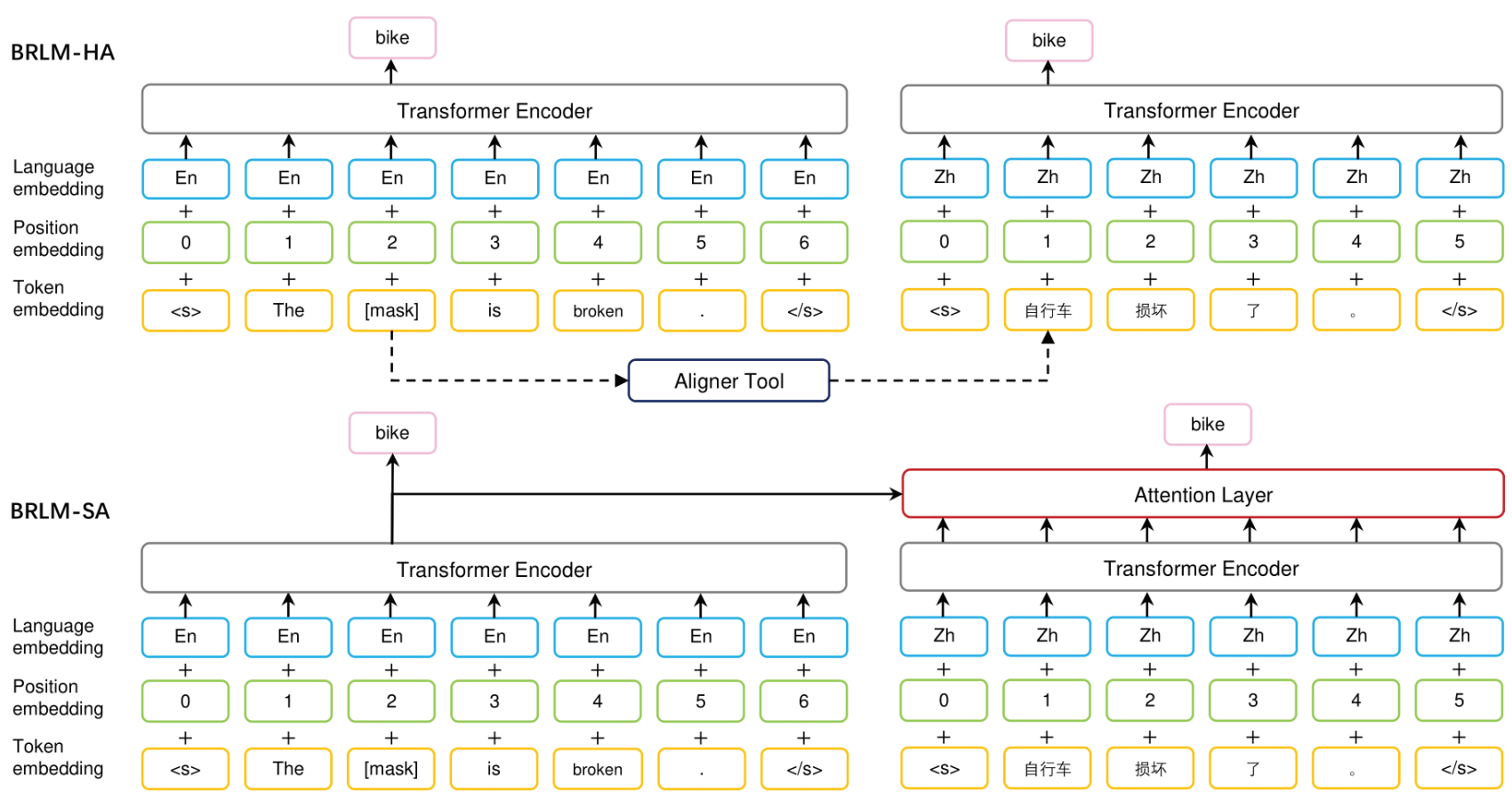

Figure 2: The overview of BRidge Language Modeling (BRLM). The BRLM extends MLM (Lample and Conneau 2019) to pairs of parallel sentences and leverages explicit alignment information obtained by external aligner tool or additional attention layer to encourage word representation alignment across different languages.

- Directly translate source sentences into target sentences with the parent model, which benefits from the availability of the universal encoder.

The key difficulty of this method is to ensure the intermediate representations of the universal encoder are language invariant. In the rest of this section, we first present two existing methods yet to be explored in zero-shot translation, and then propose a straightforward but effective cross-lingual pre-training method. In the end, we present the whole training and inference protocol for transfer.

\section{Masked and Translation Language Model Pretraining}

Two existing cross-lingual pre-training methods, Masked Language Modeling (MLM) and Translation Language Modeling (TLM), have shown their effectiveness on XNLI cross-lingual classification task (Lample and Conneau 2019; Huang et al. 2019), but these methods have not been well studied on cross-lingual generation tasks in zero-shot condition. We attempt to take advantage of the cross-lingual ability of the two methods for zero-shot translation.

Specifically, MLM adopts the Cloze objective of BERT (Devlin et al. 2018) and predicts the masked words that are randomly selected and replaced with [MASK] token on monolingual corpus. In practice, MLM takes different language monolingual corpora as input to find features shared across different languages. With this method, word pieces shared in all languages have been mapped into a shared space, which makes the sentence representations across different languages close (Pires, Schlinger, and Garrette 2019).

Since MLM objective is unsupervised and only requires monolingual data, TLM is designed to leverage parallel data when it is available. Actually, TLM is a simple extension of MLM, with the difference that TLM concatenates sentence pair into a whole sentence, and then randomly masks words in both the source and target sentences. In this way, the model can either attend to surrounding words or to the translation sentence, implicitly encouraging the model to align the source and target language representations. Note that although each sentence pair is formed into one sentence, the positions of the target sentence are reset to count form zero.

\section{Bridge Language Model Pretraining}

Aside from MLM and TLM, we propose BRidge Language Modeling (BRLM) to further obtain word-level representation alignment between different languages. This method is inspired by the assumption that if the feature spaces of different languages are aligned very well, the masked words in the corrupted sentence can also be guessed by the context of the correspondingly aligned words on the other side. To achieve this goal, BRLM is designed to strengthen the ability to infer words across languages based on alignment information, instead of inferring words within monolingual sentence as in MLM or within the pseudo sentence formed by concatenating sentence pair as in TLM.

As illustrated in Figure 2, BRLM stacks shared encoder over both side sentences separately. In particular, we design two network structures for BRLM, which are divided into 
Hard Alignment (BRLM-HA) and Soft Alignment (BRLMSA) according to the way of generating the alignment information. These two structures actually extend MLM into a bilingual scenario, with the difference that BRLM leverages external aligner tool or additional attention layer to explicitly introduce alignment information during model training.

- Hard Alignment (BRLM-HA). We first use external aligner tool on source $\leftrightarrow$ pivot parallel data to extract the alignment information of sentence pair. During model training, given source $\leftrightarrow$ pivot sentence pair, BRLM-HA randomly masks some words in source sentence and leverages alignment information to obtain the aligned words in pivot sentence for masked words. Based on the processed input, BRLM-HA adopts the Transformer (Vaswani et al. 2017) encoder to gain the hidden states for source and pivot sentences respectively. Then the training objective of BRLM-HA is to predict the masked words by not only the surrounding words in source sentence but also the encoder outputs of the aligned words. Note that this training process is also carried out in a symmetric situation, in which we mask some words in pivot sentence and obtain the aligned words in the source sentence.

- Soft Alignment (BRLM-SA). Instead of using external aligner tool, BRLM-SA introduces an additional attention layer to learn the alignment information together with model training. In this way, BRLM-SA avoids the effect caused by external wrong alignment information and enables many-to-one soft alignment during model training. Similar with BRLM-HA, the training objective of BRLMSA is to predict the masked words by not only the surrounding words in source sentence but also the outputs of attention layer. In our implementation, the attention layer is a multi-head attention layer adopted in Transformer, where the queries come from the masked source sentence, the keys and values come from the pivot sentence.

In principle, MLM and TLM can learn some implicit alignment information during model training. However, the alignment process in MLM is inefficient since the shared word pieces only account for a small proportion of the whole corpus, resulting in the difficulty of expanding the shared information to align the whole corpus. TLM also lacks effort in alignment between the source and target sentences since TLM concatenates the sentence pair into one sequence, making the explicit alignment between the source and target infeasible. BRLM fully utilizes the alignment information to obtain better word-level representation alignment between different languages, which better relieves the burden of the domain shift problem.

\section{Transfer Protocol}

We consider the typical zero-shot translation scenario in which a high resource pivot language has parallel data with both source and target languages, while source and target languages has no parallel data between themselves. Our proposed cross-lingual pretraining based transfer approach for source $\rightarrow$ target zero-shot translation is mainly divided into two phrases: the pretraining phase and the transfer phase.

\begin{tabular}{l|c|c|c|c}
\hline Corpus & Language & Train & Dev & Test \\
\hline \multirow{3}{*}{ Europarl } & De-En,En-Fr & $1 \mathrm{M}, 1 \mathrm{M}$ & 2,000 & 2,000 \\
& Fr-En,En-Es & $1 \mathrm{M}, 1 \mathrm{M}$ & 2,000 & 2,000 \\
& Ro-En,En-De & $0.6 \mathrm{M}, 1.5 \mathrm{M}$ & 2,000 & 1,000 \\
\hline \multirow{2}{*}{ MultiUN } & Ar-En,En-Es & $9.7 \mathrm{M}, 11.3 \mathrm{M}$ & 4,000 & 4,000 \\
& En-Ru & $11.6 \mathrm{M}$ & & \\
\hline
\end{tabular}

Table 1: Data Statistics.

In the pretraining phase, we first pretrain MLM on monolingual corpora of both source and pivot languages, and continue to pretrain TLM or the proposed BRLM on the available parallel data between source and pivot languages, in order to build a cross-lingual encoder shared by the source and pivot languages.

In the transfer phase, we train pivot $\rightarrow$ target NMT model initialized by the cross-lingually pre-trained encoder, and finally transfer the trained NMT model to source $\rightarrow$ target translation thanks to the shared encoder. Note that during training pivot $\rightarrow$ target NMT model, we freeze several layers of the cross-lingually pre-trained encoder to avoid the degeneracy issue.

For the more complicated scenario that either the source side or the target side has multiple languages, the encoder and the decoder are also shared across each side languages for efficient deployment of translation between multiple languages.

\section{Experiments}

\section{Setup}

We evaluate our cross-lingual pre-training based transfer approach against several strong baselines on two public datatsets, Europarl (Koehn 2005) and MultiUN (Eisele and Chen 2010), which contain multi-parallel evaluation data to assess the zero-shot performance. In all experiments, we use BLEU as the automatic metric for translation evaluation. ${ }^{1}$

Datasets. The statistics of Europarl and MultiUN corpora are summarized in Table 1. For Europarl corpus, we evaluate on French-English-Spanish (Fr-En-Es), GermanEnglish-French (De-En-Fr) and Romanian-English-German (Ro-En-De), where English acts as the pivot language, its left side is the source language, and its right side is the target language. We remove the multi-parallel sentences between different training corpora to ensure zero-shot settings. We use the devtest2006 as the validation set and the test2006 as the test set for $\mathrm{Fr} \rightarrow \mathrm{Es}$ and $\mathrm{De} \rightarrow \mathrm{Fr}$. For distant language pair Ro $\rightarrow$ De, we extract 1,000 overlapping sentences from newstest2016 as the test set and the 2,000 overlapping sentences split from the training set as the validation set since there is no official validation and test sets. For vocabulary, we use 60K sub-word tokens based on Byte Pair Encoding (BPE) (Sennrich, Haddow, and Birch 2015).

For MultiUN corpus, we use four languages: English (En) is set as the pivot language, which has parallel data

\footnotetext{
${ }^{1}$ We calculate BLEU scores with the multi-bleu.perl script.
} 


\begin{tabular}{|c|c|c|c|c|c|c|}
\hline Europarl & \multicolumn{2}{|c|}{$\mathrm{Fr} \rightarrow \mathrm{En} \rightarrow \mathrm{Es}$} & \multicolumn{2}{|c|}{$\mathrm{De} \rightarrow \mathrm{En} \rightarrow \mathrm{Fr}$} & \multicolumn{2}{|c|}{$\mathrm{Ro} \rightarrow \mathrm{En} \rightarrow \mathrm{De}$} \\
\hline Direction & Fr $\rightarrow$ Es & En $\rightarrow$ Es & $\mathrm{De} \rightarrow \mathrm{Fr}$ & $\mathrm{En} \rightarrow \mathrm{Fr}$ & $\mathrm{Ro} \rightarrow \mathrm{De}$ & En $\rightarrow$ De \\
\hline \multicolumn{7}{|c|}{ Baselines } \\
\hline Cross-lingual Transfer (Kim, Gao, and Ney 2019) & 18.45 & 34.01 & 9.86 & 34.05 & 2.02 & 23.61 \\
\hline MNMT(Johnson et al & 27.12 & 34.69 & 21.36 & 33.87 & 9.31 & 24.09 \\
\hline MNMT $_{\text {Agreement }}($ Al-Shedivat and Parikh 2019) & 29.91 & 33.80 & 24.45 & 32.55 & - & - \\
\hline Pivoting & 32.25 & 34.01 & 27.79 & 34.05 & 14.74 & 23.61 \\
\hline \multicolumn{7}{|c|}{ Proposed Cross-lingual Pretraining Based Transfer } \\
\hline MLM & 35.96 & 34.83 & 27.61 & 35.66 & 12.64 & 22.04 \\
\hline MLM+TLM & 36.78 & 34.73 & 29.45 & 35.33 & 14.39 & 24.96 \\
\hline MLM+BRLM-HA & 36.30 & 34.98 & 29.91 & 34.99 & 14.21 & 24.26 \\
\hline MLM+BRLM-SA & 37.02 & 34.92 & 30.66 & 35.91 & 15.62 & 24.95 \\
\hline
\end{tabular}

Table 2: Results on Europarl test sets. Three pivot settings are conducted in our experiments. In each setting, the left column presents the zero-shot performances (source $\rightarrow$ target), and the right column denotes the performances in the supervised parent model direction (pivot $\rightarrow$ target).

with other three languages which do not have parallel data between each other. The three languages are Arabic (Ar), Spanish (Es), and Russian (Ru), and mutual translation between themselves constitutes six zero-shot translation direction for evaluation. We use $80 \mathrm{~K}$ BPE splits as the vocabulary. Note that all sentences are tokenized by the tokenize.perl ${ }^{2}$ script, and we lowercase all data to avoid a large vocabulary for the MultiUN corpus.

Experimental Details. We use traditional transfer learning, pivot-based method and multilingual NMT as our baselines. For the fair comparison, the Transformer-big model with 1024 embedding/hidden units, 4096 feed-forward filter size, 6 layers and 8 heads per layer is adopted for all translation models in our experiments. We set the batch size to 2400 per batch and limit sentence length to $100 \mathrm{BPE}$ tokens. We set the attn_drop $=0$ (a dropout rate on each attention head), which is favorable to the zero-shot translation and has no effect on supervised translation directions $(\mathrm{Gu}$ et al. 2019). For the model initialization, we use Facebook's cross-lingual pretrained models released by $\mathrm{XLM}^{3}$ to initialize the encoder part, and the rest parameters are initialized with xavier uniform. We employ the Adam optimizer with $\mathrm{lr}=0.0001, t_{\text {warm_up }}=4000$ and dropout $=0.1$. At decoding time, we generate greedily with length penalty $\alpha=1.0$.

Regarding MLM, TLM and BRLM, as mentioned in the pre-training phase of transfer protocol, we first pre-train MLM on monolingual data of both source and pivot languages, then leverage the parameters of MLM to initialize TLM and the proposed BRLM, which are continued to be optimized with source-pivot bilingual data. In our experiments, we use MLM+TLM, MLM+BRLM to represent this training process. For the masking strategy during training, following Devlin et al. (2018), 15\% of BPE tokens are selected to be masked. Among the selected tokens, $80 \%$ of them are replaced with [MASK] token, $10 \%$ are replaced

\footnotetext{
${ }^{2}$ https://github.com/moses-smt/mosesdecoder/blob/RELEASE3.0/scripts/tokenizer/tokenizer.perl

${ }^{3}$ https://github.com/facebookresearch/XLM
}

with a random BPE token, and $10 \%$ unchanged. The prediction accuracy of masked words is used as a stopping criterion in the pre-training stage. Besides, we use fastalign tool (Dyer, Chahuneau, and Smith 2013) to extract word alignments for BRLM-HA.

\section{Main Results}

Table 2 and 3 report zero-shot results on Europarl and Multi-UN evaluation sets, respectively. We compare our approaches with related approaches of pivoting, multilingual NMT (MNMT) (Johnson et al. 2016), and cross-lingual transfer without pretraining (Kim, Gao, and Ney 2019). The results show that our approaches consistently outperform other approaches across languages and datasets, especially surpass pivoting, which is a strong baseline in the zeroshot scenario that multilingual NMT systems often fail to beat (Johnson et al. 2016; Al-Shedivat and Parikh 2019; Arivazhagan et al. 2018). Pivoting translates source to pivot then to target in two steps, causing inefficient translation process. Our approaches use one encoder-decoder model to translate between any zero-shot directions, which is more efficient than pivoting. Regarding the comparison between transfer approaches, our cross-lingual pretraining based transfer outperforms transfer method that does not use pretraining by a large margin.

Results on Europarl Dataset. Regarding comparison between the baselines in table 2, we find that pivoting is the strongest baseline that has significant advantage over other two baselines. Cross-lingual transfer for languages without shared vocabularies (Kim, Gao, and Ney 2019) manifests the worst performance because of not using source $\leftrightarrow$ pivot parallel data, which is utilized as beneficial supervised signal for the other two baselines.

Our best approach of MLM+BRLM-SA achieves the significant superior performance to all baselines in the zeroshot directions, improving by 0.9-4.8 BLEU points over the strong pivoting. Meanwhile, in the supervised direction of pivot $\rightarrow$ target, our approaches performs even better than the original supervised Transformer thanks to the shared en- 


\begin{tabular}{|c|c|c|c|c|c|c|c|c|}
\hline MultiUN & \multicolumn{8}{|c|}{$\mathrm{Ar}, \mathrm{Es}, \mathrm{Ru} \leftrightarrow \mathrm{En}$} \\
\hline Direction & $\mathrm{Ar} \rightarrow \mathrm{Es}$ & $\mathrm{Es} \rightarrow \mathrm{Ar}$ & $\mathrm{Ar} \rightarrow \mathrm{Ru}$ & $\mathrm{Ru} \rightarrow \mathrm{Ar}$ & $\mathrm{Es} \rightarrow \mathrm{Ru}$ & $\mathrm{Ru} \rightarrow \mathrm{Es}$ & A-ZST & A-ST \\
\hline \multicolumn{9}{|c|}{ Baselines } \\
\hline Cross-lingual Transfer & 10.26 & 12.44 & 4.58 & 4.42 & 13.80 & 7.93 & 8.90 & 44.73 \\
\hline MNMT(Johnson et al. 2016) & 27.40 & 20.18 & 15.12 & 16.19 & 17.88 & 27.93 & 20.78 & 43.95 \\
\hline Pivoting $_{m}$ & 42.29 & 30.15 & 27.23 & 26.16 & 29.57 & 40.08 & 32.58 & 43.95 \\
\hline \multicolumn{9}{|c|}{ Proposed Cross-lingual Pretraining Based Transfer } \\
\hline MLM & 16.50 & 23.41 & 9.61 & 14.23 & 22.80 & 23.66 & 18.36 & 44.25 \\
\hline MLM+TLM & 25.98 & 26.55 & 16.84 & 20.07 & 25.91 & 29.52 & 24.14 & 43.71 \\
\hline MLM+BRLM-HA & 29.05 & 27.58 & 18.10 & 20.42 & 25.39 & 30.96 & 25.25 & 44.67 \\
\hline MLM+BRLM-SA & 36.01 & 31.08 & 25.49 & 25.06 & 30.47 & 36.01 & 30.68 & 44.54 \\
\hline \multicolumn{9}{|c|}{ Adding Back Translation } \\
\hline MNMT* (Gu et al. 2019) & 39.72 & 28.05 & 24.67 & 24.43 & 27.41 & 38.01 & 30.38 & 43.98 \\
\hline MLM & 40.98 & 31.53 & 26.06 & 26.69 & 31.28 & 40.02 & 32.76 & 44.28 \\
\hline MLM+TLM & 41.15 & 29.77 & 27.61 & 27.74 & 31.02 & 40.37 & 32.39 & 44.14 \\
\hline MLM+BRLM-HA & 41.74 & 31.89 & 27.24 & 27.54 & 31.29 & 40.34 & 33.35 & 44.52 \\
\hline MLM+BRLM-SA & 44.17 & 33.20 & 29.01 & 28.91 & 32.53 & 41.93 & 34.95 & 45.49 \\
\hline
\end{tabular}

Table 3: Results on MultiUN test sets. The six zero-shot translation directions are evaluated. The column "A-ZST" reports averaged BLEU of zero-shot translation, while the column "A-ST" reports averaged BLEU of supervised pivot $\rightarrow$ target direction.

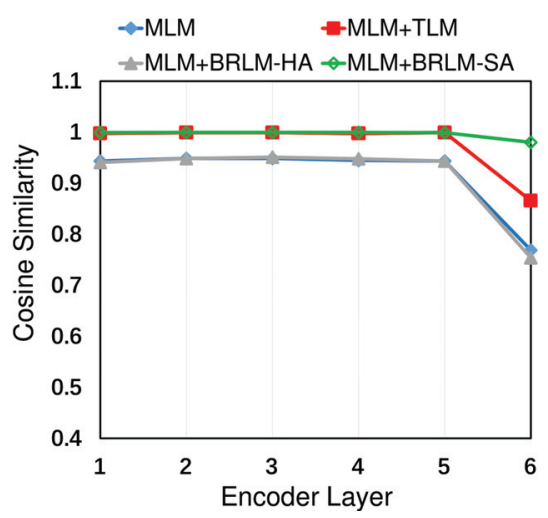

(a) Fr-En

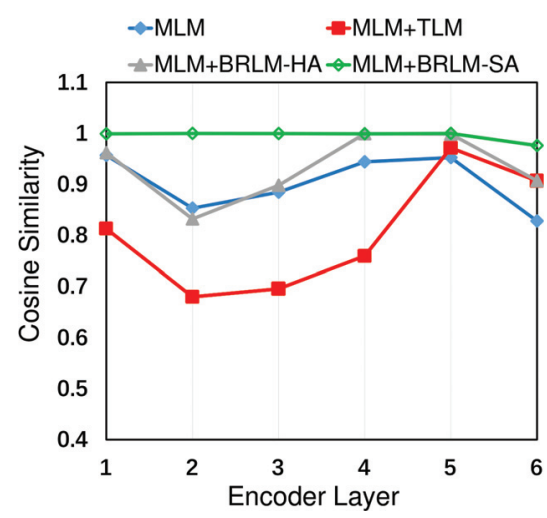

(b) De-En

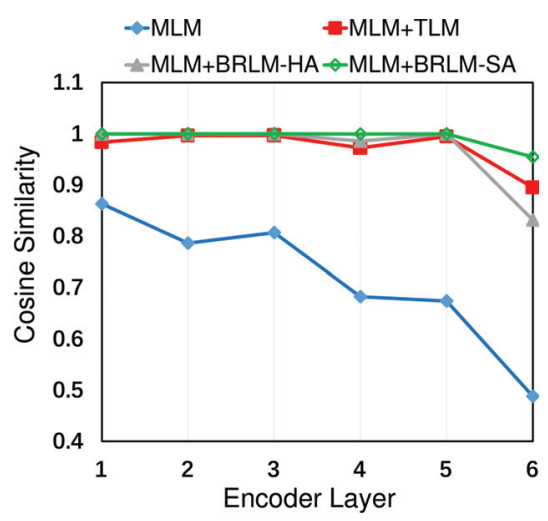

(c) Ro-En

Figure 3: Cosine similarity between sentence representation of each encoder layer across all source-pivot sentence pairs in the Europarl validation set.

coder trained on both large-scale monolingual data and parallel data between multiple languages.

MLM alone that does not use source $\leftrightarrow$ pivot parallel data performs much better than the cross-lingual transfer, and achieves comparable results to pivoting. When MLM is combined with TLM or the proposed BRLM, the performance is further improved. MLM+BRLM-SA performs the best, and is better than MLM+BRLM-HA indicating that soft alignment is helpful than hard alignment for the crosslingual pretraining.

Results on MultiUN Dataset. Like experimental results on Europarl, MLM+BRLM-SA performs the best among all proposed cross-lingual pretraining based transfer approaches as shown in Table 3. When comparing systems consisting of one encoder-decoder model for all zero-shot translation, our approaches performs significantly better than MNMT (Johnson et al. 2016).
Although it is challenging for one model to translate all zero-shot directions between multiple distant language pairs of MultiUN, MLM+BRLM-SA still achieves better performances on Es $\rightarrow \mathrm{Ar}$ and $\mathrm{Es} \rightarrow \mathrm{Ru}$ than strong pivoting ${ }_{\mathrm{m}}$, which uses MNMT to translate source to pivot then to target in two separate steps with each step receiving supervised signal of parallel corpora. Our approaches surpass pivoting in all zero-shot directions by adding back translation (Sennrich, Haddow, and Birch 2015) to generate pseudo parallel sentences for all zero-shot directions based on our pretrained models such as MLM+BRLM-SA, and further training our universal encoder-decoder model with these pseudo data. $\mathrm{Gu}$ et al. (2019) introduces back translation into MNMT, while we adopt it in our transfer approaches. Finally, our best MLM+BRLM-SA with back translation outperforms pivoting $_{m}$ by 2.4 BLEU points averagely, and outperforms MNMT (Gu et al. 2019) by 4.6 BLEU points averagely. Again, in supervised translation directions, MLM+BRLM- 
SA with back translation also achieves better performance than the original supervised Transformer.

\section{Analysis}

Sentence Representation. We first evaluate the representational invariance across languages for all cross-lingual pretraining methods. Following Arivazhagan et al. (2018), we adopt max-pooling operation to collect the sentence representation of each encoder layer for all source-pivot sentence pairs in the Europarl validation sets. Then we calculate the cosine similarity for each sentence pair and average all cosine scores. As shown in Figure 3, we can observe that, MLM+BRLM-SA has the most stable and similar cross-lingual representations of sentence pairs on all layers, while it achieves the best performance in zero-shot translation. This demonstrates that better cross-lingual representations can benefit for the process of transfer learning. Besides, MLM+BRLM-HA is not as superior as MLM+BRLMSA and even worse than MLM+TLM on Fr-En, since MLM+BRLM-HA may suffer from the wrong alignment knowledge from an external aligner tool. We also find an interesting phenomenon that as the number of layers increases, the cosine similarity decreases.

Contextualized Word Representation. We further sample an English-Russian sentence pair from the MultiUN validation sets and visualize the cosine similarity between hidden states of the top encoder layer to further investigate the difference of all cross-lingual pre-training methods. As shown in Figure 4, the hidden states generated by MLM+BRLM-SA have higher similarity for two aligned words. It indicates that MLM+BRLM-SA can gain better word-level representation alignment between source and pivot languages, which better relieves the burden of the domain shift problem.

The Effect of Freezing Parameters. To freeze parameters is a common strategy to avoid catastrophic forgetting in transfer learning (Howard and Ruder 2018). Table 4 shows the performance of transfer learning with freezing different layers on MultiUN test set, in which $\mathrm{En} \rightarrow \mathrm{Ru}$ denotes the parent model, $\mathrm{Ar} \rightarrow \mathrm{Ru}$ and $\mathrm{Es} \rightarrow \mathrm{Ru}$ are two child models, and all models are based on MLM+BRLM-SA. We can find that updating all parameters during training will cause a notable drop on the zero-shot direction due to the catastrophic forgetting. On the contrary, freezing all the parameters leads to the decline on supervised direction because the language features extracted during pre-training is not sufficient for MT task. Freezing the first four layers of the transformer shows the best performance and keeps the balance between pre-training and fine-tuning.

\section{Conclusion}

In this paper, we propose a cross-lingual pretraining based transfer approach for the challenging zero-shot translation task, in which source and target languages have no parallel data, while they both have parallel data with a high resource

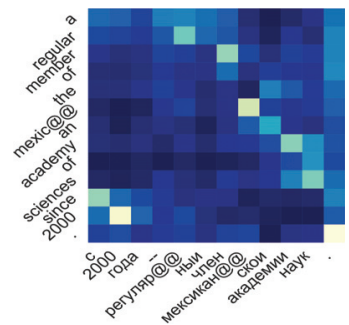

(a) MLM

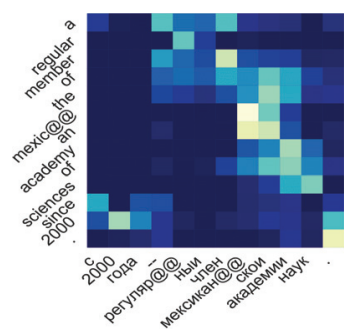

(c) MLM+BRLM-HA

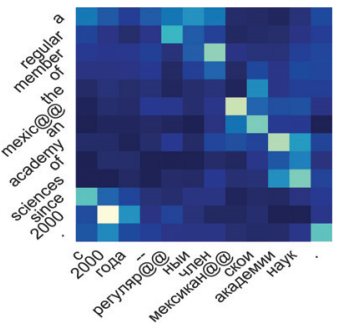

(b) MLM+TLM

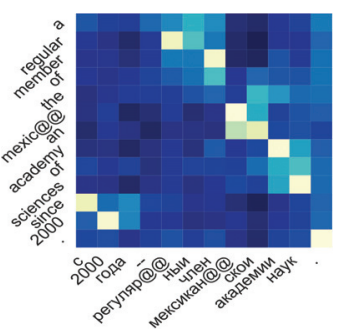

(d) MLM+BRLM-SA
Figure 4: Cosine similarity visualization at word level given an English-Russian sentence pair from the MultiUN validation sets. Brighter indicates higher similarity.

\begin{tabular}{c|c|c|c}
\hline Freezing Layers & $\mathrm{En} \rightarrow \mathrm{Ru}$ & $\mathrm{Ar} \rightarrow \mathrm{Ru}$ & $\mathrm{Es} \rightarrow \mathrm{Ru}$ \\
\hline None & 37.80 & 16.09 & 19.80 \\
2 & 37.79 & 21.47 & 28.35 \\
4 & 37.55 & $\mathbf{2 5 . 4 9}$ & $\mathbf{3 0 . 4 7}$ \\
6 & 35.31 & 22.90 & 28.22 \\
\hline
\end{tabular}

Table 4: BLEU score of freezing different layers. The number in Freezing Layers column denotes that the number of encoder layers will not be updated.

pivot language. With the aim of building the language invariant representation between source and pivot languages for smooth transfer of the parent model of pivot $\rightarrow$ target direction to the child model of source $\rightarrow$ target direction, we introduce one monolingual pretraining method and two bilingual pretraining methods to construct an universal encoder for the source and pivot languages. Experiments on public datasets show that our approaches significantly outperforms several strong baseline systems, and manifest the language invariance characteristics in both sentence level and word level neural representations.

\section{Acknowledgments}

We would like to thank the anonymous reviewers for the helpful comments. This work was supported by National Key R\&D Program of China (Grant No. 2016YFE0132100), National Natural Science Foundation of China (Grant No. $61525205,61673289)$. This work was also partially supported by Alibaba Group through Alibaba Innovative Research Program and the Priority Academic Program Development (PAPD) of Jiangsu Higher Education Institutions. 


\section{References}

Aharoni, R.; Johnson, M.; and Firat, O. 2019. Massively multilingual neural machine translation. In NAACL-HLT.

Al-Shedivat, M., and Parikh, A. P. 2019. Consistency by agreement in zero-shot neural machine translation. In NAACL-HLT.

Arivazhagan, N.; Bapna, A.; Firat, O.; Aharoni, R.; Johnson, M.; and Macherey, W. 2018. The missing ingredient in zeroshot neural machine translation. ArXiv abs/1903.07091.

Artetxe, M.; Labaka, G.; Agirre, E.; and Cho, K. 2017. Unsupervised neural machine translation. ArXiv $\mathrm{abs} / 1710.11041$.

Chen, Y.; Liu, Y. P.; Cheng, Y.; and Li, V. O. K. 2017. A teacher-student framework for zero-resource neural machine translation. In $A C L$.

de Gispert, A., and Mariño, J. B. 2006. Catalan-english statistical machine translation without parallel corpus : Bridging through spanish.

Devlin, J.; Chang, M.-W.; Lee, K.; and Toutanova, K. 2018. Bert: Pre-training of deep bidirectional transformers for language understanding. In NAACL-HLT.

Dyer, C.; Chahuneau, V.; and Smith, N. A. 2013. A simple, fast, and effective reparameterization of ibm model 2. In HLT-NAACL.

Eisele, A., and Chen, Y. 2010. Multiun: A multilingual corpus from united nation documents. In LREC.

Firat, O.; Sankaran, B.; Al-Onaizan, Y.; Yarman-Vural, F. T.; and Cho, K. 2016. Zero-resource translation with multilingual neural machine translation. In EMNLP.

Firat, O.; Cho, K.; and Bengio, Y. 2016. Multi-way, multilingual neural machine translation with a shared attention mechanism. In HLT-NAACL.

Fu, Y.; Hospedales, T. M.; Xiang, T. Y.; and Gong, S. 2015. Transductive multi-view zero-shot learning. IEEE Transactions on Pattern Analysis and Machine Intelligence 37:2332-2345.

Gu, J.; Wang, Y.; Cho, K.; and Li, V. O. K. 2019. Improved zero-shot neural machine translation via ignoring spurious correlations. In $A C L$.

Hassan, H.; Aue, A.; Chen, C.; Chowdhary, V.; Clark, J. R.; Federmann, C.; Huang, X.; Junczys-Dowmunt, M.; Lewis, W.; Li, M.; Liu, S.; Liu, T. M.; Luo, R.; Menezes, A.; Qin, T.; Seide, F.; Tan, X.; Tian, F.; Wu, L.; Wu, S.; Xia, Y.; Zhang, D.; Zhang, Z.; and Zhou, M. 2018. Achieving human parity on automatic chinese to english news translation. ArXiv abs/1803.05567.

Howard, J., and Ruder, S. 2018. Universal language model fine-tuning for text classification. In $A C L$.

Huang, H.; Liang, Y.; Duan, N.; Gong, M.; Shou, L.; Jiang, D.; and Zhou, M. 2019. Unicoder: A universal language encoder by pre-training with multiple cross-lingual tasks. ArXiv abs/1909.00964.

Johnson, M.; Schuster, M.; Le, Q. V.; Krikun, M.; Wu, Y.; Chen, Z.; Thorat, N.; Viégas, F. B.; Wattenberg, M.; Corrado, G. S.; Hughes, M.; and Dean, J. 2016. Google's mul- tilingual neural machine translation system: Enabling zeroshot translation. Transactions of the Association for Computational Linguistics 5:339-351.

Kauers, M.; Vogel, S.; Fügen, C.; and Waibel, A. H. 2002. Interlingua based statistical machine translation. In INTERSPEECH.

Kim, Y.; Petrov, P.; Petrushkov, P.; Khadivi, S.; and Ney, H. 2019. Pivot-based transfer learning for neural machine translation between non-english languages. ArXiv abs/1909.09524.

Kim, Y.; Gao, Y.; and Ney, H. 2019. Effective cross-lingual transfer of neural machine translation models without shared vocabularies. In $A C L$.

Kocmi, T., and Bojar, O. 2018. Trivial transfer learning for low-resource neural machine translation. In WMT.

Koehn, P., and Knowles, R. 2017. Six challenges for neural machine translation. In NMT@ACL.

Koehn, P. 2005. Europarl: A parallel corpus for statistical machine translation.

Lample, G., and Conneau, A. 2019. Cross-lingual language model pretraining. ArXiv abs/1901.07291.

Lample, G.; Ott, M.; Conneau, A.; Denoyer, L.; and Ranzato, M. 2018. Phrase-based \& neural unsupervised machine translation. In EMNLP.

Nguyen, T. Q., and Chiang, D. 2017. Transfer learning across low-resource, related languages for neural machine translation. In IJCNLP.

Pires, T.; Schlinger, E.; and Garrette, D. 2019. How multilingual is multilingual bert? In $A C L$.

Ren, S.; Zhang, Z.; Liu, S.; Zhou, M.; and Ma, S. 2019. Unsupervised neural machine translation with smt as posterior regularization. In $A A A I$.

Sennrich, R.; Haddow, B.; and Birch, A. 2015. Neural machine translation of rare words with subword units. In $A C L$.

Utiyama, M., and Isahara, H. 2007. A comparison of pivot methods for phrase-based statistical machine translation. In HLT-NAACL.

Vaswani, A.; Shazeer, N.; Parmar, N.; Uszkoreit, J.; Jones, L.; Gomez, A. N.; Kaiser, L.; and Polosukhin, I. 2017. Attention is all you need. In NIPS.

Wu, Y.; Schuster, M.; Chen, Z.; Le, Q. V.; Norouzi, M.; Macherey, W.; Krikun, M.; Cao, Y.; Gao, Q.; Macherey, K.; et al. 2016. Google's neural machine translation system: Bridging the gap between human and machine translation. arXiv preprint arXiv:1609.08144.

Zheng, H.; Cheng, Y.; and Liu, Y. P. 2017. Maximum expected likelihood estimation for zero-resource neural machine translation. In IJCAI.

Zhu, X.; He, Z.; Wu, H.; Wang, H.; Zhu, C.; and Zhao, T. 2013. Improving pivot-based statistical machine translation using random walk. In EMNLP.

Zoph, B.; Yuret, D.; May, J.; and Knight, K. 2016. Transfer learning for low-resource neural machine translation. In EMNLP. 